\title{
EFICACIA INICIAL DE UNA INTERVENCIÓN SOCIO-COGNITIVA PARA PADRES Y MADRES DE NIÑOS Y NIÑAS DE DESCENDENCIA PUERTORRIQUEÑA CON CONDUCTAS DE ALTO RIESGO
}

\author{
Coralee Pérez Pedrogo' \\ Eduardo Cumba Áviles ${ }^{2}$ \\ Sean K. Sayers ${ }^{3}$
}

\section{Resumen}

Evaluamos la eficacia inicial de una intervención sociocognitiva para padres y madres de niños y niñas de descendencia puertorriqueña con conductas de alto riesgo. Participaron 18 madres de niños y niñas entre las edades de 5 a 12 años. Administramos el Inventario de Experiencia Familiar y el Inventario de Prácticas de Crianza antes y después de la intervención. Encontramos un aumento en las prácticas de crianza adecuadas y una disminución en las inadecuadas a raíz de la intervención $(p<.01)$. Además, las madres reportaron una disminución del estrés familiar global $(p<.01)$, del estrés relacionado al ambiente escolar $(p<.01)$, de los sentimientos negativos hacia sus hijos e hijas $(p<.05)$ y del estrés asociado a la relación de pareja $(p<.05)$. Los hallazgos apoyan la eficacia inicial de la intervención. Discutimos las implicaciones y limitaciones de los resultados. [Descriptores: Intervención socio-cognitiva, prácticas de crianza, conductas de alto riesgo].

\section{Abstract}

We evaluated the initial efficacy of a socio-cognitive intervention for parents of children of Puerto Rican ancestry with high risk behaviors. Participants were 18 mothers of children aged

\footnotetext{
Médicas

'Psicóloga Clínica, Universidad de Puerto Rico, Recinto de Ciencias Médicas

${ }^{2}$ Psicólogo Clínico, Universidad de Puerto Rico, Recinto de Ciencias

${ }^{3}$ Psicólogo Clínico, Universidad Carlos Albizu
} 
5 to 12 years. We administered the Family Experiences Inventory and the Parent Practices Inventory before and after treatment. We found an increase in the adequate parent practices and a reduction in the inadequate parent practices at post-treatment $(p<.01)$. Moreover, the mothers reported further reductions in global family stress $(p<.01)$, in the stress related to the school environment $(p<.01)$, in their negative feelings toward her children $(p<.05)$, and in the stress associated to couple relationship. The results support the initial efficacy of the intervention. We discussed our findings implications and limitations. [Key Words: Socio-cognitive intervention, parent practices, high risk behaviors].

La familia es un escenario crucial en el desarrollo infantil y la principal responsable por la crianza. La crianza es un proceso complejo mediante el cual el niño y la niña hace suyos aquellos hábitos, valores, expectativas y patrones de conducta que le son transmitidos por los padres, las madres y la sociedad. Definimos las prácticas de crianza como las estrategias a través de las cuales los padres y las madres ayudan a sus hijos e hijas en el proceso de socialización.

Es importante considerar la influencia cultural al dar cuenta de los procesos de crianza. Salas (2003) señala que los valores, los hábitos, las expectativas y los patrones de conducta apropiados son definidos por el grupo social en el cual las personas se desenvuelven. Éste define en cierta medida la conducta esperada por el niño y la niña y los patrones de crianza que utilicen los padres y las madres en el manejo de esa conducta. En el caso de la crianza de los niños y las niñas que son de descendencia hispana, ésta hace énfasis en el control parental y en la importancia del afecto por parte del niño y de la niña, la búsqueda de la cercanía física, el respeto a la autoridad parental, la obediencia, la lealtad a la familia y el honor personal (FríasArmenta y McCloskey, 1998; Zayas y Solari, 1994). Las prácticas de crianza pueden tener significados diferentes dependiendo de la cultura en la que se practiquen. Ejemplo de ello es el castigo físico, que en algunas culturas es visto como estrategia de disciplina y no como una práctica de agresión física (FríasArmenta y McCloskey, 1998).

Las prácticas empleadas con los niños y las niñas juegan un rol determinante en el proceso de crianza. En el plano emocional, los niños y las niñas son recipientes directos de las emociones negativas o positivas de los padres y las madres y de la familia en general (Salas, 2003). El grado en que los niños y las niñas están expuestos y expuestas a prácticas de crianza punitivas, inconsistentes y agresivas puede estar relacionado con el desarrollo, el mantenimiento y la intensidad de los problemas 
de comportamiento (Abidin, 1992; Frick, Christian y Wootton, 1999; Stormshack, Bierman, McMaon y Lengua, 2000), lo que a su vez puede contribuir a un aumento en el estrés familiar percibido por los padres y las madres con relación a esa conducta (Salas, 2003).

Múltiples investigaciones han identificado las prácticas de crianza como un factor de riesgo para el desarrollo de la conducta perturbadora en niños y niñas (Brody y Ge, 2001; Getz y Bray, 2005; Huebner y Howell, 2003; Miller, 2002; Van den Bree y Pickworth, 2005). La categoría de conductas de alto riesgo, según es utilizada en este estudio, incluye desorden de conducta, impulsividad, agresividad, hostilidad y el desorden oposicionaldesafiante, el cual constituye el mayor problema mental infantil, afectando cerca del $10 \%$ de los niños y las niñas de escuela elemental (Kazdin, 1987). Un estudio epidemiológico realizado en Puerto Rico reflejó una prevalencia de $12.6 \%$ para cualquier tipo de conducta perturbadora, $8.9 \%$ para el Trastorno por Déficit de Atención con Hiperactividad (TDAH) y $6.0 \%$ para el Trastorno Oposicional Desafiante (TOD) (Canino et al., 2004). Además, la categoría de las conductas de alto riesgo anteriormente descrita está asociada con el uso de drogas, el abuso de alcohol, la violencia familiar, los crímenes, así como con varios trastornos psiquiátricos (Brody y Ge, 2001; Getz y Bray, 2005; Huebner y Howell, 2003; Miller, 2002; Van den Bree y Pickworth, 2005).

Las investigaciones que estudian la relación entre los patrones de crianza y las conductas de alto riesgo en los niños y las niñas se enfocan mayormente en el TOD y en la conducta agresiva. Rothbaum y Weiz (1994) realizaron un meta-análisis de 47 estudios en el cual examinaron las prácticas de crianza y la conducta disruptiva. Deseaban analizar qué tipos de prácticas de crianza eran las más confiables para predecir esta conducta (agresividad, hostilidad, desobediencia, entre otras). Desarrollaron seis categorías de variables de crianza, definiéndolas como aprobación (reforzar lo positivo), dirección (supervisar y proveer mensajes claros y consistentes), estrategias de motivación (incentivos), sincronía (prestar atención y escuchar), control coercitivo (intentar influenciar al niño y a la niña con el uso de la fuerza o repitiendo mandatos) y restricción (poner límites). Al reanalizar los resultados de los estudios vistos desde el punto de vista de las variables individuales de crianza, notaron que las variables de aprobación, dirección, estrategias para la motivación, sincronía y ausencia de control coercitivo estaban negativamente asociadas con la conducta disruptiva. En otras palabras, a mayor presencia de las variables antes descritas, menor era la presencia de conducta disruptiva en el niño y la niña. 
Diversos estudios han identificado una asociación entre informes retrospectivos de uso de prácticas inconsistentes (por ejemplo, negligencia excesiva por parte de la madre) y las conductas de alto riesgo (delincuencia y agresión) en los niños y las niñas (Dix, 1991; Frías-Armenta y McCloskey 1998). Frick, Christian y Wootton (1999) encontraron que los padres y las madres de niños y niñas que exhiben agresividad, desobediencia y presentan rasgos antisociales utilizan intensos intercambios coercitivos con sus hijos e hijas y tienden a emplear prácticas más sumisas, ambiguas e inconsistentes en sus respuestas a los problemas de comportamiento. Además, ellos y ellas tienden a reforzar comportamientos oposicionales con atención y no son consistentes ni firmes dando sus mandatos. Otros estudios han demostrado que la escasa involucración y supervisión diaria de progenitores hacia su prole durante la niñez contribuyen a problemas de agresión y violencia en la adolescencia (Farrington, 1991). Por otro lado, niños y niñas con progenitores que proveen estructura y supervisión diaria durante las horas de estudio tienden a no presentar problemas de conducta y a tener un mayor rendimiento académico (Deslandes y Royer, 1997). La falta de comunicación entre los padres y las madres hacia sus hijos e hijas, al igual que la escasa supervisión puede contribuir a los desórdenes conductuales en los niños y en las niñas de nivel escolar.

Utilizando diseños que permiten inferencias causales, diversos investigadores $e$ investigadoras han manipulado algunos parámetros de disciplina y han examinado los efectos de esas variables en el comportamiento de niños y niñas. Estudios realizados con niños y niñas de nivel preescolar y elemental con problemas indicaron que cuando sus padres y madres reemplazan la disciplina inadecuada con prácticas de crianza más claras, firmes, consistentes y apropiadas, sus hijos e hijas exhiben menos agresividad (Dishion, 1998; Stormshack, Bierman, Mc Maon y Lengua, 2000). Strayhorn y Weidman (1998) han ofrecido sugerencias para el manejo de conducta de los niños y las niñas. Algunas de ellas han sido proveer un ambiente físico agradable, reducir la necesidad de órdenes, reconocer y apreciar cualquier trabajo que el niño y la niña realice, estimular su intelecto y habilidades, y evitar a toda costa castigos físicos. Esto con miras a reducir las conductas de alto riesgo, evitando el uso de prácticas de crianza inadecuadas e incrementando la frecuencia con que utilizan las adecuadas.

En dos estudios previos en los que participó la primera autora se identificaron diversas dimensiones en las prácticas utilizadas por las madres que pueden contribuir a que los niños 
y las niñas presenten conductas de alto riesgo (Pérez-Pedrogo y Matos, 2001, 2002). Algunas de ellas fueron la inconsistencia, el uso de mandatos indirectos, los pocos refuerzos y la poca demostración de afecto cuando los padres y las madres tienen el control. Las autoras concluyeron que la intervención temprana es necesaria para identificar y tratar a tiempo aquellas prácticas o estrategias utilizadas para el manejo de la conducta que pueden ser contraproducentes.

Algunos estudios han demostrado que las terapias manualizadas de corte cognitivo- conductual son efectivas para niños y niñas con conductas de alto riesgo (Brestan y Eyberg, 1998; Kazdin, 2003; Lochman y Wells, 2003; Lochman, Wells y Lenhart, 2008; Miranda y Presentación, 2000; Reid, Stratton y Hammond, 2003). El Programa Coping Power (CPP, por sus siglas en inglés) es una intervención socio-cognitiva manualizada diseñada para reducir los problemas de conducta (Lochman, Wells y Lenhart, 2008). Siguiendo un marco evolutivo, este modelo conceptualiza las conductas de alto riesgo como el resultado de varios factores familiares y personales, siendo la conducta agresiva de los niños y las niñas parte del desarrollo del problema. Según Lochman, Wells y Murria (en imprenta) los niños y las niñas que presentan conductas agresivas experimentan reacciones negativas de parte de sus padres, madres, maestros, maestras y pares que hacen que sus lazos con la escuela se debiliten y que estén vulnerables a involucrarse con grupos de pares que muestren conductas socialmente desviadas.

El CPP consta de tres componentes: (1) niño y niña; (2) padre y madre; (3) maestro y maestra (Lochman, Wells y Lenhart, 2008). El componente de los padres y las madres consta de 16 talleres grupales de 90 minutos de duración. EI mismo está basado en un modelo de aprendizaje social y provee destrezas para el manejo de la agresión en hijos e hijas. Además, provee orientación sobre cómo dar atención y refuerzo positivo por la conducta apropiada. La intervención ofrece adiestramiento acerca de cómo ignorar conductas disruptivas, cómo proveer reglas y cómo transmitir expectativas claras. También enfatiza la consistencia de las consecuencias negativas por la conducta inapropiada de parte del niño y de la niña (ejemplo, retiro de privilegios), estrategias de solución de problemas, comunicación familiar, expresión de sentimientos y manejo del estrés (tanto en los aspectos físicos como emocionales). Finalmente, este programa ofrece estrategias para ofrecer apoyo escolar en el hogar. Lochman y Wells (2003) demostraron la eficacia de esta intervención reduciendo las conductas agresivas y otras conductas de alto riesgo en niños y niñas con desórdenes disruptivos. 
Dada la alta prevalencia de las conductas disruptivas y perturbadoras en la población infantil con descendencia puertorriqueña, y la falta de tratamientos validados para niños y niñas y sus familias, surge la necesidad de evaluar la eficacia de una intervención para padres y madres de niños y niñas con descendencia puertorriqueña entre las edades de 5 a 12 años que exhiban conductas de alto riesgo. En este estudio se pretende desarrollar, implantar y evaluar la eficacia inicial de una intervención psicosocial breve basada en el CPP desarrollado por Lochman y Wells (2003), pero tomando en consideración el contexto sociocultural puertorriqueño. Este tipo de intervención ha mostrado ser efectiva para la población anglosajona en la reducción de conductas de alto riesgo. Sin embargo, no se han realizado estudios con miras a examinar su eficacia en nuestro contexto.

Postulamos dos hipótesis fundamentales. La primera propone que se observarán diferencias estadísticamente significativas $(p<.05)$ en las prácticas de crianza empleadas por las madres entre la medida pre- y pos-tratamiento. Específicamente, se observará un incremento en el uso de prácticas positivas, y una disminución en las negativas, una vez finalizada la intervención. La segunda hipótesis supone que las madres reportarán una reducción significativa en el nivel global de estrés familiar entre la medida pre- y pos-tratamiento.

\section{Método}

\section{Participantes}

Participaron 18 madres de niños y niñas entre las edades de 5 a 12 años que cursaban grados de primero a sexto en una escuela pública de Puerto Rico. Los niños y las niñas presentaban problemas de conducta, impulsividad y/o agresividad. Otros criterios de inclusión fueron los siguientes: ausencia de dificultades sensoriales, neurológicas, en el lenguaje o en el desarrollo; no tener retraso mental, no estar recibiendo otros servicios psicológicos durante la intervención, ni presentar ideación suicida. Los niños representaron el 78 \% (14), mientras que las niñas el $22 \%$ (4) de la muestra. Dos terceras partes de los niños y las niñas tenían entre 5 y 8 años. Su edad promedio fue de 7.78 años. El 50\% (9) de las madres reportó un nivel educativo de escuela superior, un $33 \%$ (6) dijo tener estudios subgraduados y un 5.6 (1) reportó sólo un nivel educativo de escuela elemental. Dos de las madres no proveyeron esta información.

\section{Instrumentos}

Inventario de Comportamiento Escuela (IDC-E) - Este inventario, completado por los maestros y las maestras, evalúa 
síntomas de ansiedad, retraimiento social, depresión, irritabilidadhostilidad, distracción-motivación y actividad-impulsividad. En el caso de la subescala de Actividad-Impulsividad, su consistencia interna (alpha) es de .95 para los varones y de .93 para las niñas. La consistencia interna de la subescala de IrritabilidadHostilidad es de .94 y .91 para varones y niñas, respectivamente. Los valores de confiabilidad temporal para ambos géneros en las escalas utilizadas fluctúan entre .77 y .89 (Bauermeister, 1994).

Inventario de Prácticas de Crianza (IPC) - EI IPC fue desarrollado en el 1995 por Bauermeister, Salas y Matos, usando algunos reactivos del Parent Practices Scales (Strayhorn y Weidman, 1988) y del Parenting Scale (Arnold, O'Leary, Wolff y Acker, 1993). Los mismos fueron traducidos al español, adaptados culturalmente y revisados tomando en consideración las prácticas de crianza de los padres y las madres de descendencia puertorriqueña. También se construyeron reactivos adicionales con base en la experiencia clínica de los autores y las autoras. En este estudio se utilizó una versión del IPC que consta de 37 reactivos (Barkley, Murphy y Bauermeister, 1998) que la madre evalúa en una escala que fluctúa de 0 (nunca o casi nunca) a 3 (muy frecuentemente). El mismo provee una puntuación total que evalúa la calidad global de las prácticas de crianza y contiene varios reactivos para medir la frecuencia con que los padres y las madres utilizan prácticas de crianza inadecuadas. La consistencia interna y la confiabilidad temporal (intervalo de 4 a 6 semanas) del IPC son de .85 al utilizarse para evaluar las prácticas de crianza de las madres de los niños y las niñas en edad escolar (Bauermeister, Matos, Reina, Salas, Martínez, Cumba, y Barkely, 2005).

Inventario de Experiencia Familiar (IEF). EI IEF también fue diseñado en el 1995 por Bauermeister, Matos y Salas. Consta de ocho escalas diseñadas para evaluar cómo la conducta del niño y de la niña en la escuela y en el hogar afecta los sentimientos de progenitores hacia su prole, la vida social de la familia, los costos del hogar, las relaciones con la escuela, las preocupaciones acerca del futuro del niño y de la niña, las relaciones con hermanos y hermanas y las relaciones con la pareja y (si aplica) el padre o la madre que no viva en el hogar (Bauermeister et al., 2005). La puntuación total utilizada en este estudio se obtiene al sumar las puntuaciones de las primeras cinco áreas. La consistencia interna y la confiabilidad temporal del IEF han demostrado ser altas (.93), y tiene también una excelente validez de constructo. 


\section{Procedimiento}

\section{Fase 1: Pre tratamiento}

Se solicitó por medio de carta oficial al Director Escolar la participación y la autorización para llevar a cabo el estudio. Se llevó a cabo una reunión para explicarle el propósito, la población a impactar y los criterios de inclusión y exclusión en la selección de la muestra. Obtenida la autorización del Director Escolar, se realizaron reuniones individuales con el Trabajador Social Escolar y con los maestros y las maestras con el propósito de ofrecerle información similar. Una vez obtenida la autorización del Trabajador Social Escolar y de los maestros y las maestras para llevar a cabo el estudio, el Trabajador Social Escolar contactó personalmente a los padres y a las madres de los niños y las niñas entre las edades de 5 a 12 años (primero a sexto grado). Se les informó a los padres y a las madres los días en los cuales la primera autora iba a estar disponible para aclarar dudas.

Se ofrecieron reuniones individuales a los padres y a las madres en las que se explicó el propósito del estudio y los criterios de inclusión para participar. Además se les informó acerca de la naturaleza confidencial de la información, siempre y cuando no hubiese evidencia de peligrosidad para su vida y/o la de terceras personas. Las madres interesadas firmaron una hoja de consentimiento accediendo a participar en el estudio. El presente estudio obtuvo el consentimiento del Comité de Derechos Humanos (IRB, por sus siglas en inglés) de la Universidad Carlos Albizu para poder llevar a cabo los procesos pertinentes.

Para seleccionar las madres participantes, los maestros y las maestras de sus hijos e hijas completaron el IDC-E como parte del proceso de cernimiento. Los niños y las niñas de las madres seleccionadas cumplieron con el punto de corte establecido para el perfil de impulsividad y/o agresividad. Es decir, los varones arrojaron puntuaciones $\geq 34$ en la escala de Actividad/Impulsividad y/o $\geq 24$ en la escala de Irritabilidad/Hostilidad. Las niñas arrojaron puntuaciones $\geq 29 \mathrm{y} / 0 \geq 23$ en dichas escalas, respectivamente.

Una vez seleccionadas las madres participantes, se recopiló información sociodemográfica para obtener las características descriptivas de la muestra. Además cada madre participante completó el IPC y el IEF.

Fase 2: Implantación del Tratamiento

Las madres seleccionadas fueron invitadas a asistir a seis talleres grupales de aproximadamente 90 minutos de duración, a razón de un taller por semana. En dichos talleres (basados en el CPP) se fomentaron discusiones de temas como el manejo de estrés, la disciplina, el establecer lazos con la escuela, entre otros. 
Los mismos se ofrecieron en un salón designado especialmente para este estudio, de modo que se protegiera la privacidad de las participantes. A continuación se presenta una descripción del contenido de las sesiones.

Descripción de la intervención

Sesiones 1-2. Se presenta una introducción general del programa y se discute el tema del estrés y sus manifestaciones a nivel físico y emocional y en las prácticas de crianza. Se exploran las estrategias utilizadas por las participantes en el manejo diario del estrés. Se discuten varias estrategias de corte conductual (ejemplo, relajación muscular progresiva). En estas sesiones se promueve el juego de roles y la retroalimentación por parte de la terapeuta y las participantes.

Sesión 3. En ella se presentan los conceptos básicos de la Teoría de Aprendizaje Social (ejemplo, identificación del evento, conducta y consecuencia). Otro objetivo de la sesión es desarrollar estrategias de resolución de problemas (adiestramiento en el Para, Respira y Piensa). Se introduce la importancia de identificar y reforzar en los niños y las niñas las conductas positivas.

Sesiones 4-5. El objetivo general de estas sesiones es desarrollar estrategias para manejar las conductas inapropiadas de los niños y las niñas: (a) la técnica de ignorar para conductas disruptivas menores; (b) el establecimiento de reglas e instrucciones efectivas; (c) la importancia de la disciplina efectiva y el castigo (otro distinto al físico); (d) la remoción de privilegios y (e) la asignación de tareas para aquellas conductas desadaptativas mayores que no deben ser ignoradas. En estas sesiones se fomentó la participación de las integrantes del grupo a través de los juegos de roles y la retroalimentación por parte de la terapeuta y las participantes.

Sesión 6. En ella se refuerzan las estrategias discutidas en sesiones previas a través de situaciones presentadas por las madres, discusión grupal y juego de roles dirigido a desarrollar apoderamiento en la crianza de sus hijos e hijas. Por último, se discute la importancia de la cohesión familiar como medida de atajar problemas presentes y futuros (p. ej., el uso de sustancias). Fase 3: Periodo de Pos-evaluaciones

Finalizados los seis talleres, se realizó un periodo de pos-evaluaciones a todas las participantes. Este periodo de posevaluaciones se realizó de manera individual para monitorear el progreso de las participantes. El periodo de pos-evaluaciones se ofreció en un salón de la escuela designado especialmente para este estudio y así fomentar la privacidad de las participantes. EI mismo tuvo una buena iluminación y no hubo ruidos que pudiesen 
afectar o interrumpir la administración de los instrumentos. Este periodo de pos-evaluaciones se llevó a cabo en el mismo horario designado para el periodo de pre-evaluaciones.

\section{Análisis de datos}

Primero realizamos análisis descriptivos para tener una caracterización demográfica de la muestra. De las 18 madres participantes sólo una abandonó el estudio. El análisis estadístico seleccionado para poner a prueba nuestras hipótesis fue la $t$ de muestras pareadas, utilizando la pre- y pos-prueba tanto del IPC como del IEF. El nivel de significancia utilizado fue de $p<.05$. Dicho análisis tuvo como objetivo examinar la eficacia inicial de la intervención a través de su efecto en las prácticas de crianza empleadas por las madres y el nivel de estrés familiar reportado por ellas. Analizamos los datos utilizando el Paquete Estadístico para las Ciencias Sociales (SPSS, por sus siglas en inglés).

\section{Resultados}

Los resultados mostraron una diferencia significativa $(p<.01)$ en los promedios entre las medidas pre- y posintervención en la puntuación total del IPC y en la subescala de Prácticas Inadecuadas (Vea Tabla1 en Apéndice). Las madres reportaron un aumento en las prácticas de crianza adecuadas y una disminución en las inadecuadas tras la intervención.

Por otro lado, los resultados con relación al estrés familiar reflejaron una diferencia altamente significativa en los promedios de las medidas pre- y pos-intervención en el nivel global de estrés familiar $(p<.01)$ y en la subescala relacionada al comportamiento del niño o de la niña en la escuela $(p<.01)$. Específicamente, las madres reportaron una disminución del estrés familiar global y del estrés asociado al ambiente escolar. También encontramos diferencias significativas $(p<.05)$ en otras subescalas, como la que evaluaba los sentimientos negativos de la madre respecto a la conducta de su hijo o hija, así como el impacto de dicha conducta en la relación de pareja. De modo similar, en ambas áreas el estrés reportado por las madres disminuyó tras el tratamiento. Aunque presente, el efecto de la intervención sobre el estrés reportado por las madres en la relación con los hermanos del niño o de la niña y en la vida social de la familia no alcanzó el nivel de significancia requerido ( $p>.05<10)$.

\section{Discusión}

El propósito de este estudio fue evaluar la eficacia inicial de una intervención socio-cognitiva breve para padres y madres de niños y niñas con descendencia puertorriqueña de nivel escolar con conductas de alto riesgo. Dicha evaluación se enfocó en el impacto de la misma en las prácticas de crianza 
utilizadas por las madres participantes y en el nivel de estrés familiar reportado por éstas con relación a la conducta de sus hijos e hijas. Conforme a nuestra primera hipótesis, encontramos diferencias significativas en las prácticas de crianza empleadas por las madres entre la medida pre-tratamiento y la medida pos-intervención Rechazamos, por tanto, la hipótesis nula al encontrarse tanto un aumento en la calidad global de las prácticas de crianza como una disminución significativa en las prácticas de crianza negativas que las madres reportaron utilizar.

En los análisis relacionados a la segunda hipótesis, donde esperábamos encontrar diferencias en el nivel global de estrés familiar entre las medidas de pre y pos tratamiento, los resultados obtenidos mostraron diferencias estadísticamente significativas en los promedios no sólo del nivel global de estrés familiar, sino también de la subescalas relacionadas al comportamiento del niño o de la niña en la escuela, a los sentimientos de la madre y a la relación de pareja. Las madres reportaron una disminución estadísticamente más significativa en el estrés familiar global y en el asociado al ámbito escolar, sugiriendo una posible disminución en la intensidad en los problemas de conducta infantiles presentados tanto en la escuela como en el hogar, o al menos que el incremento en la capacidad materna para manejar adecuadamente las conductas de su prole (es decir, su mejoría en las prácticas de crianza) resultó en una merma en el estrés o malestar psicológico asociado a la crianza de sus hijos e hijas con problemas de conducta. Los resultados apoyan los estudios correlacionales realizados con niños y niñas de descendencia puertorriqueña de edad escolar (Bauermeister et al, 1996; Pérez-Pedrogo y Matos, 2001), donde a mayor uso de prácticas de crianza adecuadas (y menor uso de inadecuadas), menor presencia e intensidad de los problemas de conducta y menor estrés familiar asociado a éstos.

Se destaca en este estudio la importancia de enseñar a las madres cómo hacer buen uso de las prácticas de crianza. Es necesario promover en las familias una relación positiva entre madre-hijo e hija. De igual modo es crucial que las madres disminuyan las prácticas de crianza que puedan dañar esta relación. Ambas cosas sin duda están vinculadas a una disminución en los niveles de estrés familiar. La intervención socio-cognitiva breve empleada en este estudio ha demostrado ser inicialmente efectiva para lograr estos propósitos. Aunque es deseable que en toda familia los cuidadores y las cuidadoras practiquen estos principios de crianza, disciplina y atmósfera familiar, es notable y prometedor que la intervención evaluada haya resultado preliminarmente efectiva con una población de 
madres de niños y niñas con conductas de alto riesgo. Esto debido a que los padres y las madres de niños y niñas con conductas de alto riesgo están más propensos a utilizar con mayor frecuencia, prácticas de crianza inadecuadas y con menor frecuencia las prácticas adecuadas (Abidin, 1992, Routhbaum y Weisz, 1994; Salas, 2003; Strayhorn y Weidman, 1998). Asi también estas familias tienden a tener ambientes familiares que se asocian a un mayor estrés que las familias de niños y niñas que no presentan estas conductas (Bauermeister et al., 2005; Salas, 2003). Podría suponerse, pues, que la intervención sería potencialmente efectiva para padres y madres de niños y niñas de la población general que no presenten estas conductas, debido a la menor severidad en los problemas relacionados a las áreas mencionadas.

Indudablemente son múltiples los factores que inciden en el origen y desarrollo de los problemas de conducta en niños y niñas. Los factores de riesgo pueden incluir variables específicas del niño o de la niña, variables del padre y/o de la madre, la dinámica interaccional intrafamiliar y en la comunidad, el estilo de crianza y las variables sociodemográficas, entre otros (Salas, 2003). La evaluación de la presencia o ausencia de estos factores debe ser considerada en forma individual, de manera que el tratamiento ofrecido sea más efectivo, acomodándose a la necesidades de cada familia (Lolas, 1991).

Son diversas las recomendaciones a sugerirse a partir de este estudio. Ya que es un estudio piloto, es importante continuar realizando investigaciones más rigurosas y amplias que documenten la eficacia de la intervención socio-cognitiva utilizada. En las mismas se debe incluir un número mayor de participantes, para incrementar el poder estadístico de los análisis para detectar cambios significativos. Para aumentar la capacidad de generalizar el hallazgo inicial de eficacia de esta intervención, también sería conveniente ofrecer los talleres a grupos de padres de niños y niñas con conductas de alto riesgo, así como a grupos donde se combinen madres y padres. De igual modo, sería conveniente hacer dicho estudio en escenarios de escuela pública vs. escuela privada. Por otro lado, para mejorar la validez interna de estudios futuros, sería importante tener un grupo control comparable al que reciba la intervención, de modo que los resultados se puedan adjudicar más directamente, y de manera más concluyente, a la misma.

Es pertinente incluir como parte del protocolo medidas que permitan evaluar otras áreas importantes no incluidas en este estudio. Ejemplo de ello podrían ser instrumentos como el Eyberg Child Behavior Inventory (Eyberg y Pincus, 1999) que permiten 
evaluar si efectivamente la intervención ayuda a disminuir la frecuencia con que se presentan las conductas de alto riesgo en niños y niñas de padres y madres que participan de los talleres, y si la percepción de éstas personas de cuán problemática es dicha conducta mejora tras la intervención. Debe considerarse, además, un tiempo de al menos 3 meses para re-evaluar y observar si se mantienen los resultados del tratamiento a corto y a largo plazo. La administración de sesiones de seguimiento (booster sessions) es otra recomendación pertinente para lograr obtener resultados más contundentes que incrementen la evidencia a favor de la eficacia de esta intervención breve. El presente estudio representa un primer paso en dicha dirección. Agradecimientos

Se agradece la colaboración del Dr. José J. Cabiya en la selección de la muestra y por sus recomendaciones en el diseño del estudio. 


\section{Referencias}

Abidin, Richard. (1992). The determinants of parenting behavior. Journal of Clinical Child Psychology, 21, 407-412.

Arnold, David S., O' Leary, Susan G., Wolff, Lisa S. y Acker, Maureen M. (1993). The Parenting Scale: A measure of dysfunctional parenting in discipline situations. Psychological Assessment, 5, 137-144.

Barkley, Russell. A., Murphy, Kevin y Bauermeister, José J. (1998). Trastorno por déficit de atención con hiperactividad: Un manual de trabajo clínico. New York: Guilford.

Bauermeister, José J. (1994). Desarrollo y utilización del Inventario de Comportamiento Escuela (IDC-E) en la evaluación de niños puertorriqueños. San Juan, Puerto Rico: Publicaciones Puertorriqueñas.

Bauermeister, José J., Matos, Maribel, Reina, Grace, Salas, Carmen C., Martínez, José V., Cumba, Eduardo y Barkely, Russell A. (2005). Comparison of the DSM-IV combined and inattentive types of ADHD in a school-based sample of Latino/Hispanic children. Journal of Child Psychology and Psychiatry, 46, 166179.

Bauermeister, José J., Salas, Carmen C., Matos, Maribel, Reina, Grace, Ávila, Dyhalma, Martínez, José V. y Bernal, Guillermo (1996, November). Measures of Parenting Practices Inventory and Family Experiences Scale. Poster session presented at the $8^{\text {th }}$ CHADD Annual Convention, Chicago, Illinois.

Brestan, Elizabeth \& Eyberg, Sheila (1998). Effective psychosocial treatments of conduct-disordered children and adolescents: Twenty nine years, 82 studies \& 5, 272 kids. Journal of Clinical Child Psychology. 27, 180-189.

Brody, Gene H. y Ge, Xiaojia. (2001). Linking parenting processes and self-regulation to psychological functioning and alcohol use during early adolescence. Journal of Family Psychology, 15, 82-94.

Canino, Glorisa, Shrout, Patrick E., Rubio-Stipec, Maritza, Bird, Héctor R., Bravo, Milagros, Ramírez, Rafael, Chavez, Ligia, Alegría, Margarita, Bauermeister, José, Hohmann, Ann, Ribera, Julio, García, Pedro y Martínez Taboas, Alfonso. (2004). The DSM-IV rates of child and adolescent disorders in Puerto Rico. Archives of General Psychiatry, 61, 85-93.

Deslandes, Rollande y Royer, Egide (1997). Family-related variables and school disciplinary events at the secondary level. Behavioral Disorders, 23, 18-28.

Dix, Theodore. (1991). The affective organization of parenting adaptive and maladaptive processes. Psychological Bulletin, 110, 3-25.

Dishion, Thomas J. y McMahon, Robert J. (1998). Parental monitoring and the prevention of child and adolescent problem behavior: $\mathrm{A}$ conceptual and empirical formulation. Clinical Child and Family Psychology Review, 1, 3-21. 
Eyberg, Shiela y Pincus, Donna (1999). ECBI and SESBI-R (Eyberg Child Behavior Inventory and Sutter-Eyberg Student Behavior Inventory-Revised). Miami, U.S.: Psychological Assessment Resources, Inc.

Farrington, David P. (Ed.) (1991). Childhood aggression and adult violence. Hillsdale, NJ: Erlbaum.

Frías-Armenta, Martha y McCloskey, Laura A. (1998). Determinants of harsh parenting in Mexico. Journal of Abnormal Child Psychology, 26, 129-139.

Frick, Paul J., Christian, Rachel E. y Wootton, Jane M. (1999). Age trends in the association between parenting practices and conduct problems. Behavior Modification, 23, 106-128.

Getz, J. Greg y Bray, James H. (2005). Predicting heavy alcohol use among adolescents. American Journal of Orthopsychiatry, 75, 102-116.

Huebner, Angela J. y Howell, Laurie W. (2003). Examining the relationship between adolescent sexual risk-taking and perceptions of monitoring, communication, and parenting skills. Journal of Adolescent Health, 33, 71-78.

Kazdin, Alan E. (1987). Conduct disorder in childhood and adolescence. Newbury Park, CA: Sage.

Kazdin, Alan E. (2003). Problem-solving skills training and parent management training for conduct disorder. In Alan E. Kazdin y John R. Weisz (Eds.), Evidence-based psychotherapies for children and adolescents (pp. 241-261). New York, U.S.: Guilford Press.

Lochman, John y Wells, Karen. (2003). Effectiveness of the Coping Power Program and of classroom intervention with aggressive children: Outcomes at a 1-year follow-up. Behavior Therapy, 34, 493-515.

Lochman, John, Wells, Karen y Lenhart, Lisa A. (2008). Coping Power: Child Group Facilitator's Guide. New York: Oxford University Press.

Lochman, John E, Wells, Karen y Murray, Maureen (In press). The Coping Power Program: Preventive intervention and the middle school transition. In Patrick. Tolan, José Szapocznic y Soledad Sambrano (Eds.), Preventing substance abuse: Three to 14. Washington, D.C.: American Psychological Association.

Lolas, Fernando. (1991). Agresividad, agresión, violencia. En Fernando Lolas (Compilador). Buenos Aires: Losada, S.A.

Miller, Brent (2002). Family influences on adolescent sexual and contraceptive behavior. Journal of Sex Research, 39, 22-26.

Miranda, Ana y Presentación, María J. (2000). Efficacy of cognitivebehavioral therapy in the treatment of children with ADHD, with and without aggressiveness. Psychology in the Schools, 37, 169-182.

Pérez-Pedrogo, Coralee y Matos, Maribel (2001, abril). Prácticas de crianza en familias puertorriqueñas de niños/as de nivel escolar y su relación con los problemas de conducta. Presentación en el Décimo Simposio de las Ciencias Sociales, Mayagüez, PR. 
Pérez-Pedrogo, Coralee y Matos, Maribel. (2002). Prácticas de crianza en familias

puertorriqueñas de niños/as de nivel preescolar y su relación con los problemas de conducta. Manuscrito sin publicar, Universidad de Puerto Rico en Río Piedras.

Reid, Jamila, Webster-Stratton, Carolyn y Hammond, Mary. (2003). Follow-up of children who received the incredible year intervention for Oppositional-Defiant Disorder: Maintenance and prediction of 2-year outcome. Behavior Therapy, 34, 471-491.

Routhbaum, Fred, y Weisz, John R. (1994). Parental care giving and child externalizing behavior in nonclinical samples: A meta-analysis. Psychological Bulletin, 116, 55-74.

Salas, Carmen. (2003). La crianza de los niños y las niñas con el trastorno por déficit de atención con hiperactividad y su entorno familiar y social. Disertación doctoral, Universidad de Puerto Rico, Río Piedras, 2001. Dissertation Abstracts International, 64, 1505.

Stormshack, Elizabeth, Bierman, Karen, Mc Maon, Robert y Lengua, Lilliana. (2000).

Parenting practices and child disruptive behavior problems in early elementary school. Journal of Clinical Child Psychology, 29, 17-29.

Strayhorn, Joseph M. y Weidman, Carla S. (1998). A parent practices scale and its relation to parent and child mental health. Journal of the American Academy of Child \&Adolescent Psychiatry, 27, 613-618.

Van den Bree, Marianne B. M. y Pickworth, Wallace B. (2005). Risk factors predicting changes in marijuana involvement in teenagers. Archives of General Psychiatry, 62, 311-319.

Zayas, Luis H. y Solari, Fabiana. (1994). Early childhood socialization in Hispanic families: Context, culture, and practice implications. Professional Psychology: Research and Practice, 25, 200-206. 


\section{Apéndice}

Tabla 1

Efectividad de la intervención y su efecto en las prácticas de crianza y en el estrés familiar

\begin{tabular}{|c|c|c|c|c|c|c|}
\hline \multirow[t]{2}{*}{ Medida } & \multicolumn{2}{|c|}{ Pre-Tratamiento } & \multicolumn{2}{|c|}{ Pos-Tratamiento } & \multirow[t]{2}{*}{$t$} & \multirow[t]{2}{*}{ Sig. } \\
\hline & Prom. & $D E$ & Prom. & $D E$ & & \\
\hline $\begin{array}{l}\text { Prácticas de } \\
\text { Crianza Total }\end{array}$ & 71.42 & 11.46 & 79.47 & 13.90 & -3.36 & .004 \\
\hline $\begin{array}{l}\text { Prácticas } \\
\text { Inadecuadas }\end{array}$ & 11.35 & 5.04 & 7.71 & 4.04 & 3.07 & .007 \\
\hline $\begin{array}{l}\text { Estrés Familiar } \\
\text { Total }\end{array}$ & 36.75 & 13.78 & 29.31 & 13.86 & 3.57 & .003 \\
\hline $\begin{array}{l}\text { Sentimientos de la } \\
\text { madre }\end{array}$ & 15.41 & 6.66 & 11.47 & 6.66 & 2.86 & .011 \\
\hline $\begin{array}{l}\text { Vida Social de la } \\
\text { familia }\end{array}$ & 4.76 & 3.78 & 3.53 & 3.37 & 1.75 & .099 \\
\hline Ambiente escolar & 11.47 & 3.73 & 9.41 & 3.50 & 3.06 & .007 \\
\hline Costos incurridos & 2.94 & 4.28 & 3.29 & 4.43 & -.43 & .670 \\
\hline $\begin{array}{l}\text { Preocupaciones } \\
\text { acerca del futuro }\end{array}$ & 3.19 & 3.27 & 2.50 & 3.03 & 1.24 & .233 \\
\hline $\begin{array}{l}\text { Relaciones con los } \\
\text { hermanos }\end{array}$ & 5.47 & 3.04 & 3.80 & 2.11 & 1.89 & .080 \\
\hline Relación de Pareja & 5.19 & 4.74 & 4.31 & 3.66 & 2.21 & .043 \\
\hline $\begin{array}{l}\text { Relación con el } \\
\text { Padre Biológico }\end{array}$ & 2.56 & 2.88 & 1.44 & 1.94 & 1.17 & .276 \\
\hline
\end{tabular}

Nota. Prom. = Promedio; DE = Desviación Estándar; Sig. = Nivel de significancia; $t=$ Prueba $t$ para grupos pareados; $n=9$ para Relación con el Padre Biológico; $n=15$ para Relaciones con los hermanos; $n=16$ para Preocupaciones acerca del futuro, Relación de Pareja y Estrés Familiar Total; $n=17$ para el resto de las escalas. 
